\title{
Delayed multiple intracranial aneurysms caused by left atrial myxoma: a case report and literature review
}

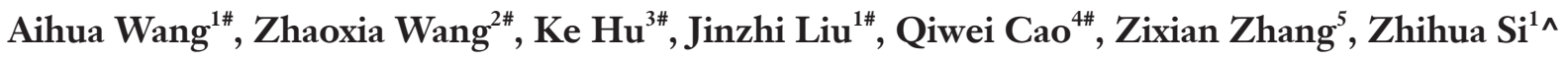 \\ ${ }^{1}$ Department of Neurology, The First Affiliated Hospital of Shandong First Medical University \& Shandong Provincial Qianfoshan Hospital, \\ Shandong Institute of Neuroimmunology, Shandong Key Laboratory of Rheumatic Disease and Translational Medicine, Jinan, China; ${ }^{2}$ Department \\ of Neurology, Juxian People's Hospital, Rizhao, China; ${ }^{3}$ Department of Emergency Medicine, The First Affiliated Hospital of Shandong First \\ Medical University \& Shandong Provincial Qianfoshan Hospital, Shandong Medicine and Health Key Laboratory of Emergency Medicine, Jinan, \\ China; ${ }^{4}$ Department of Pathology, The First Affiliated Hospital of Shandong First Medical University \& Shandong Provincial Qianfoshan Hospital, \\ Shandong Medicine and Health Key Laboratory of Clinical Pathology, Shandong Lung Cancer Institute, Shandong Institute of Nephrology, Jinan, \\ China; ${ }^{5}$ Department of Neurology, Zaozhuang Municipal Hospital, Zaozhuang, China \\ "These authors contributed equally to this work. \\ Correspondence to: Zhihua Si, MD. Department of Neurology, the First Affiliated Hospital of Shandong First Medical University \& Shandong \\ Provincial Qianfoshan Hospital, No. 16766 Jinshi Rd, Lixia District, Jinan 250014, China. Email: sizhihua35@126.com.
}

\begin{abstract}
Intracranial aneurysm may appear even after the removal of the cardiac myxoma. However, the pathogenesis and treatment of such aneurysm lesions are not clear. The study aimed to explore the clinical and imaging manifestation, hypothetical pathogenesis, and therapy in one case of left atrial myxoma causing multiple intracranial aneurysms. A 14-year-old male displayed a 3-hour history of episodic loss of consciousness and right hemiplegia after a leapfrog-like movement. The myxoma was diagnosed by a combination of clinical examination, leading to the diagnosis of mitral dynamic obstruction with a Grade III mitral diastolic murmur and tumor plop; magnetic resonance imaging, revealing multiple ischemic sites in both semi-oval centers; and transthoracic echocardiography, demonstrating a mitral valve obstruction. The myxoma was removed surgically; however, computed tomography angiography showed multiple intracranial aneurysms in both middle cerebral arteries 18 months after resection of the atrial myxoma. After conservative treatment, the patient had no neurological dysfunction symptoms for 5 years after myxoma resection. His condition is relatively stable. In conclusion, resection of the atrial myxoma may eliminate the early neurological symptoms, but it cannot ensure the nonoccurrence of delayed intracranial aneurysms. The neoplastic process theory was favored for explaining the aneurysm development in this case. According to the specific conditions of the patient, a combination of open surgery, chemotherapy, radiotherapy, and coil embolization is recommended.
\end{abstract}

Keywords: Multiple intracranial aneurysms; left atrial myxoma; magnetic resonance imaging (MRI); computed tomography angiography (CTA); case report

Submitted Jan 09, 2021. Accepted for publication Nov 29, 2021.

doi: $10.21037 / \mathrm{tp}-21-11$

View this article at: https://dx.doi.org/10.21037/tp-21-11

\footnotetext{
^ ORCID: 0000-0002-9888-3504.
} 


\section{Introduction}

Atrial myxomas are the most frequent primary benign tumors derived from the heart, and more than $80 \%$ of them are left atrial myxomas (1-3). No less than $45 \%$ of left atrial myxomas have initial clinical cardiac symptoms caused by systemic embolization. Other possible initial symptoms are neurological complications, including cerebral embolism. However, delayed neurological complications, such as multiple intracranial aneurysms, are rather rare. To date, many reports of myxoma-associated aneurysms (including intracranial and other arteries) have been published, most of which occur before myxoma resection. By contrast, a few studies have reported delayed intracranial aneurysms after myxoma resection (4). The pathogenesis of aneurysmal lesions is also not well known, and no definitive treatment has been established for them. We present the following case in accordance with the CARE reporting checklist (available at https://tp.amegroups.com/article/ view/10.21037/tp-21-11/rc).

\section{Case presentation}

All procedures performed in studies involving human participants were in accordance with the ethical standards of the institutional and/or national research committee(s) and with the Helsinki Declaration (as revised in 2013). Written informed consent was obtained from the patient's legal guardian/next of kin for publication of this case report and accompanying images. A copy of the written consent is available for review by the editorial office of this journal.

A 14-year-old male displayed a 3-hour history of episodic loss of consciousness and right hemiplegia after a leapfroglike movement, and he was admitted to Shandong Provincial Qianfoshan Hospital. His symptoms disappeared after admission. His medical history revealed that he had suffered from a cold a week before, but no familial history was found. A clinical examination demonstrated a Grade III mitral diastolic murmur and tumor plop, leading to the diagnosis of mitral dynamic obstruction. Magnetic resonance imaging (MRI) revealed multiple ischemic sites in both semi-oval centers (Figure 1A). Brain magnetic resonance angiography (MRA) did not show any abnormalities (Figure $1 B$ ). However, transthoracic echocardiography showed a large, dumbbell-shaped mass in the left atrium measuring $7 \times 5 \mathrm{~cm}^{2}$, part of the mass was protruding into the left ventricle and causing mitral valve obstruction. The ejection fraction was $69 \%$, and mild mitral and tricuspid regurgitation was observed. Taking the examination results together, the mass appeared to be a myxoma.

Following this determination, several studies were performed to reveal the pathology. No abnormalities were evident in the electrocardiography and clinical immunology studies. Laboratory tests showed no remarkable changes except for an increase in the erythrocyte sedimentation rate (37.0; normal range, $0-15 \mathrm{~mm} / \mathrm{h}$ ) and the hypersensitive C-reactive protein concentration ( $>11.10$; normal range, $0-2.87 \mathrm{mg} / \mathrm{L})$. The tumor was successfully removed by surgery and confirmed by pathology to be a myxoma (Figure 1C). The patient was discharged without postoperative symptoms or any specific treatment.

Two months after discharge, the patient was rehospitalized due to intermittent right-sided weakness. As with the first admission, the brain MRI showed ischemic lesions in the left basal ganglia and both semi-oval centers, but the brain MRA showed no abnormalities. The patient was subjected to supportive therapy and discharged in good condition.

Eighteen months after the surgery, the patient underwent computed tomography angiography (CTA), and the results revealed the presence of multiple saccular aneurysms in the distal part of the intracranial arteries (>8). Most of these aneurysms were less than $3 \mathrm{~mm}$ in size, but one dilated and the fusiform aneurysm was detected on the M3 segment of each middle cerebral artery (MCA; Figure $2 A$ ). MRI results showed infarcts of the left basal ganglia, as well as a hyperintense lesion in the anterior insula beneath the lacuna in the basal ganglia (Figure 2B). This may have been caused by a small amount of oozing of the myxomaassociated aneurysm, as the craniocerebral CTA revealed the largest aneurysm in the left middle cerebral artery. The patient underwent cardiac ultrasound 3 months after surgery and every year thereafter, and there was no evidence of recurrence of myxoma (Figure 3). The current followup results showed that the patient's condition was relatively stable and no symptoms of neurological impairment occurred 5 years after the surgery. We will continue to follow up in the future.

\section{Discussion}

Cerebral aneurysms often appear after myxoma resection for a considerable period, even years, as reported in previous cases (5). The present case showed no formation of intracranial aneurysms before the myxoma surgery, but multiple intracranial aneurysms were found 18 months after 

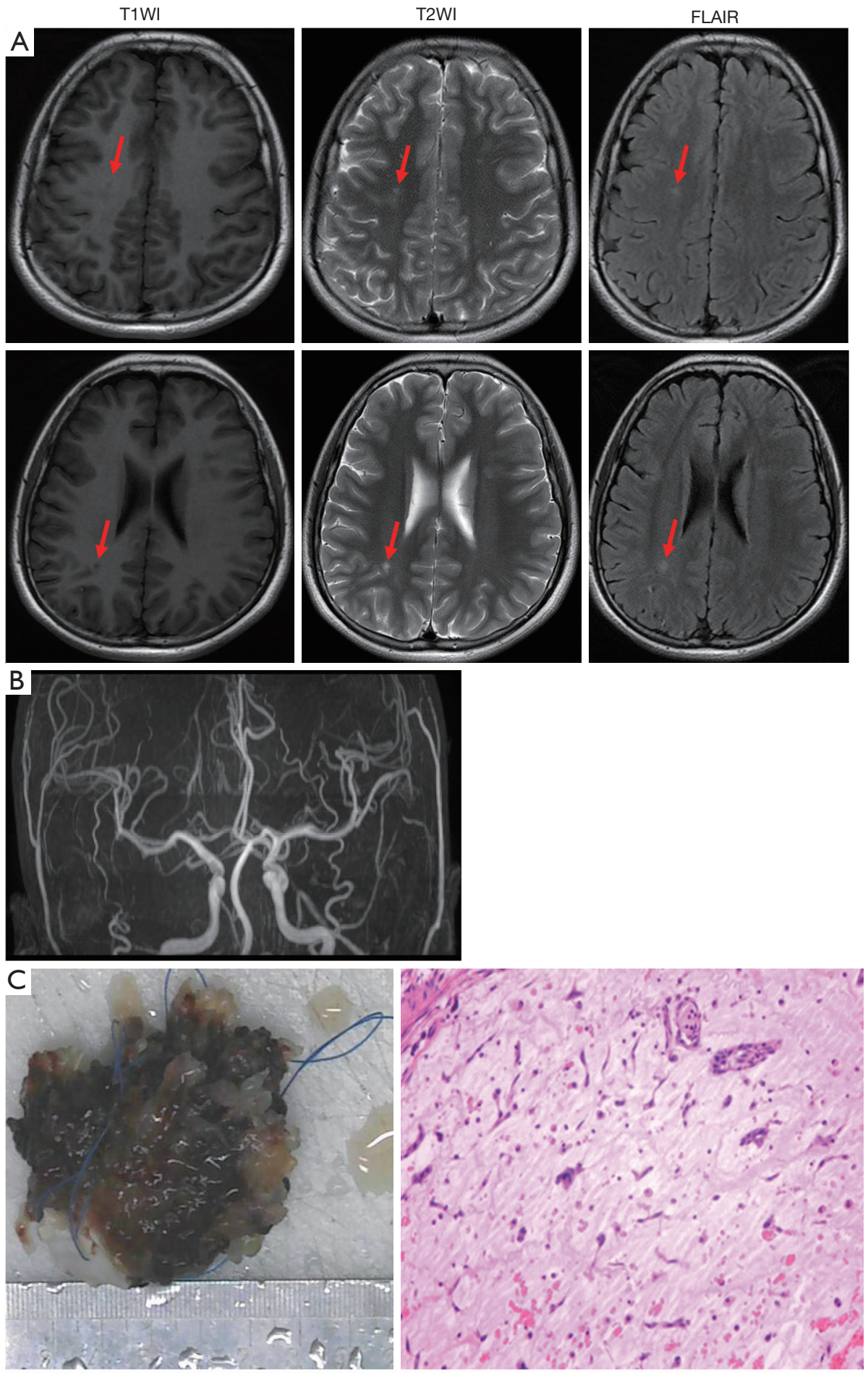

Figure 1 The examination results of the patient after admission. (A) MRI showed multiple ischemic sites (arrows) in both semi-oval centers; (B) brain MRA did not show any abnormality; (C) the tumor was confirmed to be a myxoma by hematoxylin-eosin staining (magnification: $\times 100)$. MRI, magnetic resonance imaging; MRA, magnetic resonance angiography. 

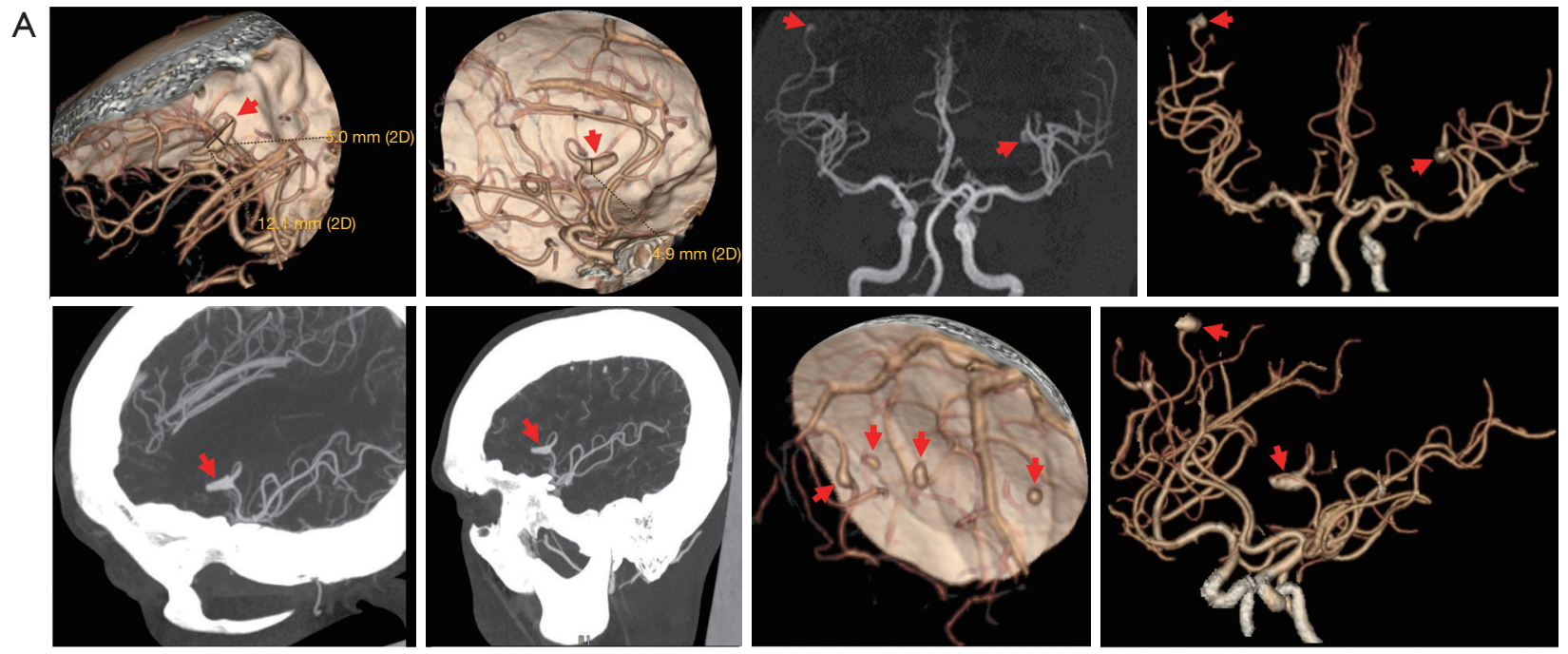

B
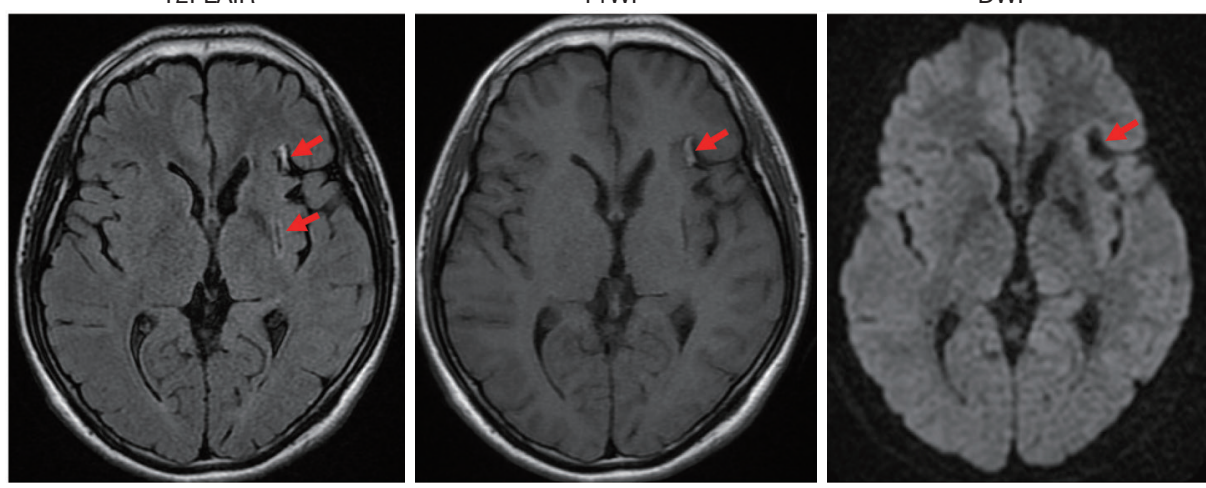

Figure 2 The examination results of the patient 18 months after myxoma resection. (A) One dilated and fusiform aneurysm was found on the M3 segment of each MCA, as well as numerous fusiform dilatations of the distal intracranial arteries, as revealed by subtraction CTA. Arrows showed the dilated and fusiform aneurysms as well as fusiform dilatations of the intracranial artery. (B) Gliotic lesions of the infarction (arrows) were observed in the left basal ganglia, along with some hyperintense lesions (arrows) in the anterior insula as revealed by MRI. MCA, middle cerebral artery; CTA, computed tomography angiography; MRI, magnetic resonance imaging.

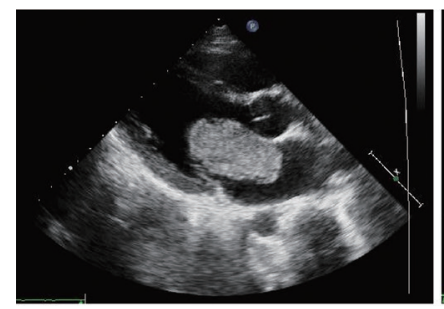

Before surgery

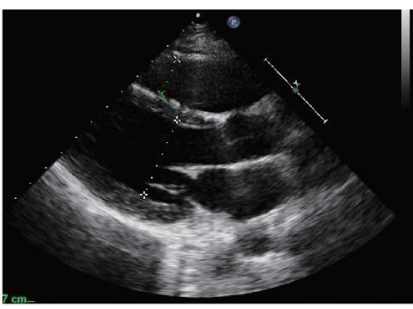

3 months after surgery

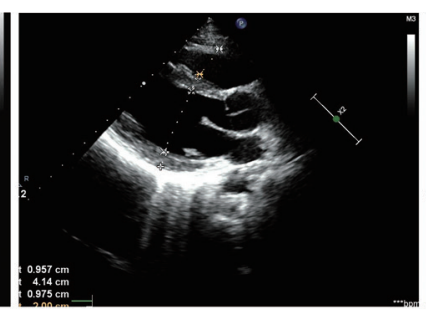

1 year after surgery

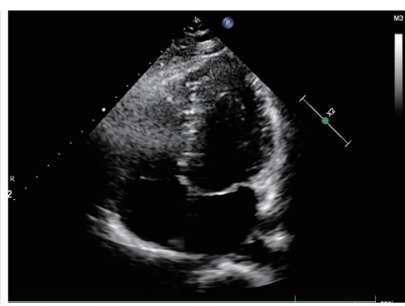

5 years after surgery

Figure 3 The cardiac ultrasound examination results before and after surgery. 
the myxoma surgery. The purpose of this case report was to remind clinicians that patients with cardiac myxoma require follow-up and monitoring for the development of brain aneurysms, even after surgical resection.

Atrial myxoma represents $50 \%$ of all primary cardiac neoplasms (6), but up to $10 \%$ of atrial myxoma patients are asymptomatic (7). The symptoms of cardiac myxomas usually include heart failure and cerebral and peripheral embolism, as well as general symptoms like fever, an elevated erythrocyte sedimentation rate, and leukocytosis (8). The release of interleukin-6 (IL-6) from the tumor cells has been proposed to account for these symptoms (6). Although these are rare, they may be caused by tumor recurrence, progressive vascular stenosis, or aneurysm development (9). A common symptom in patients with left atrial myxoma is systemic embolism, which occurs in $30-50 \%$ of cases, while cerebral embolism is involved half the time (1). Systemic emboli can lead to cerebral ischemia, subarachnoid and intraparenchymal hemorrhage, and, less commonly, the formation of aneurysms (10-12). In the present case, MRI showed multiple sites with ischemic lesions that were believed to be caused by systemic embolism. One important point to emphasize is that atrial myxomas can account for various neurological complications, even without cardiac symptoms. Intracranial aneurysms caused by a cardiac myxoma may emerge after removal of the primary tumor and can lead to delayed neurological complications at 4-5 months and even up to 25 years after resection of the cardiac mass $(13,14)$. Literature about the risk of cerebral myxomatous aneurysm rupture is scarce due to the rarity of cases. In a study by Zheng et al., in 58 patients with intracranial aneurysms, the incidence of rupture within 11 years was $19.6 \%$ (15). In the present case, the typical imaging features of these aneurysms were consistent with intracranial aneurysms following left atrial myxoma and other possible causes of aneurysms had been excluded; therefore, our patient was diagnosed with multiple intracranial aneurysms secondary to myxoma. These aneurysms are generally fusiform or saccular in shape (10) and our patient showed aneurysms with both shapes.

Aneurysms are generally distributed in the distal part of the middle cerebral arteries (MCAs). MRI and computed tomography (CT) scans can demonstrate multiple small infarcts, cerebral hemorrhages, or cerebral infarction. T1and T2-weighted MRI can reveal myxomatous aneurysms as fusiform dilatations of the cerebral arteries, and the vascular dilatation is often accompanied by edema and hemorrhage (16). Contrast enhancement of aneurysmal lesions may be secondary to the slow flow inside the lumen of fusiform aneurysms $(17,18)$. However, Nucifora et al. (17) demonstrated that this enhancement could also result from the enhancement of the tumor tissues within the aneurysm walls. MRA alone cannot exclude myxomatous aneurysms because most of them are distributed in the distal part of the circle of Willis. Therefore, the diagnosis of myxomatous aneurysms requires CTA using a high-resolution CT scanner or conventional digital subtraction angiography (DSA). On CT, aneurysms appear hyperintense, which could be secondary to the accumulation of the myxoid matrix within the aneurysm walls (19). With angiography, the most sensitive vascular imaging method, myxomatous aneurysms usually generate filling defects and delay the passage of the contrast medium $(17,20)$. In the present case, the CTA results revealed the presence of multiple saccular aneurysms, mostly less than $3 \mathrm{~mm}$ in size, in the distal part of the intracranial arteries $(>8)$, in addition to one dilated and fusiform aneurysm on the M3 segment of each MCA.

The mechanisms that give rise to these aneurysms have not been well studied, but two hypotheses have been put forward to explain their development. First, the vascular damage theory, proposes that an embolism causes vascular damage and endothelial dysfunction, thereby leading to a change in hemodynamics and promoting aneurysm development (21). By contrast, the neoplastic process theory hypothesizes that tumor cells survive and intrude into the endothelium, thereby triggering subintimal growth and eventually destroying the entire arterial vascular wall $(21,22)$. Both of these hypotheses presuppose that the embolism starts the whole course (23).

At present, researchers have tended to focus on the neoplastic process theory, since myxoma cells found in the aneurysmal wall have been shown to actively intrude into the internal elastic lamina $(11,21,22,24,25)$. However, a myxoma cannot immediately destroy the arterial vascular wall because of its slow growth (12) Consequently, myxoma cell-induced inflammation may be critical for this invasion. The release of IL-6 from tumor cells could enhance the expression and activities of matrix metalloproteinases (MMPs) (26), which are essential for the degradation of the extracellular matrix, and accordingly invasion. The IL-6 serum levels could therefore be helpful for monitoring the development of aneurysms.

Myxoma cells can proliferate in the vessel wall with or without apoptosis (27). In the former case, apoptosis in the myxoma tumor located in the arterial wall would lead to the widening of the arterial lumen and the formation of a 
fusiform aneurysm (28). In the latter non-apoptotic case, the mass would grow progressively within the intracavity, resulting in occlusion of the cerebral vessel and subsequent infarction. Under some circumstances, the formation of cerebral aneurysms may be found months and even years after the resection of myxomas in the absence of atrial myxoma recurrence (4). The cerebral aneurysms can later expand, remain stable, or regress $(4,27,29,30)$.

No definite guidelines have yet been established for therapy of multiple intracranial aneurysms following left atrial myxoma. Surgical treatment can eliminate the early neurological symptoms caused by atrial myxoma, but it cannot ensure the nonoccurrence of delayed intracranial aneurysms. The results from chemotherapy have been equivocal (31), but evidence has shown that the combination of radiotherapy and chemotherapy is useful for the treatment of myxoma metastasis, thereby reducing the likelihood of formation of myxomatous aneurysms (32-34). Other investigators have suggested that despite independent reports of microsurgical resection/endovascular intervention, chemotherapy, and radiotherapy for the treatment of cardiac myxomatous intracranial aneurysms, there is still a lack of clear guidelines (35). In this case, the presence of intracranial aneurysm associated with cardiac myxomatosis and a small amount of bleeding from the left middle cerebral artery aneurysm suggested that there might be irreversible damage to the artery wall structure. We considered a surgical clamp/ interventional embolization, but the parents refused due to concerns about the risk of surgery. As reported by a previous study, myxomatosis-associated aneurysm hemorrhage had a self-limiting process, and the amount of bleeding was generally small. In addition, the surgical treatment itself may also lead to the continued metastasis of related mucous tissue to the distal vascular implantation (36). Spontaneous regression of aneurysms has also been reported in patients who had undergone the removal of atrial myxomas $(4,27,37)$. A study of 37 published cases of myxomatous aneurysms showed that of $78.4 \%$ of patients who received conservative treatment, $75.7 \%$ showed stability or regression in followup imaging studies (30). Therefore, conservative treatment of unruptured myxomatous aneurysms with continuous angiographic follow-up (initially 3 months and later 6-12 months depending on aneurysm stability) is recommended (15). The management of other small multiple intracranial aneurysms is controversial. In patients with asymptomatic stably sized fusiform aneurysms, noninvasive imaging is recommended and aspirin can be used to prevent ischemic events, according to some investigators (38). If an aneurysm ruptures or becomes enlarged, treatment should be decided on a case-by-case basis. Atrial myxomainduced strokes are usually found in the MCA (18). Strokes secondary to cardiac myxoma can be treated safely and effectively by mechanical thrombectomy (39). This may also be the first-line treatment for pediatric stroke caused by atrial myxomas (40). By contrast, myxoma-associated aneurysms are varied and usually involve the distal vessels of the brain. Both open microsurgery and intravascular techniques have limitations that may not clear aneurysms completely, leading to further invasion and recurrence $(14,41,42)$. In our case, the current follow-up results showed that the patient's condition was relatively stable and no symptoms of neurological impairment occurred 5 years after the surgery. We will continue to follow up in the future.

In conclusion, the intracranial aneurysm caused by atrial myxoma is not a particularly common neurological complication, but it can emerge even after the removal of a cardiac myxoma. The neoplastic process theory may best explain the mechanism underlying the development of this aneurysm. MRI, CT, CTA, and DSA are the main means of diagnosis; however, no guidelines have been established for effective aneurysm treatment. Combined therapy involving open surgery, chemotherapy, radiotherapy, and coil embolization is beneficial. Patients with multiple intracranial aneurysms, and especially patients of young age, should have their history of cardiac myxoma reviewed. By the same token, patients with atrial myxoma should be monitored for the possibility of myxoma-induced intracranial aneurysms even after surgery.

\section{Acknowledgments}

Funding: This study was supported by grants from the Traditional Chinese Medicine Science and Technology Development Program of Shandong Province, China (No. 2019-0369), the Natural Science Foundation of Shandong Province, China (No. ZR2019MH062), and the Key R\&D Program of Shandong Province, China (Public Welfare) (No. 2019GSF108008 and No. 2019GSF108033).

\section{Footnote}

Reporting Checklist: The authors have completed the CARE reporting checklist. Available at https://tp.amegroups.com/ article/view/10.21037/tp-21-11/rc

Conflicts of Interest: All authors have completed the ICMJE 
uniform disclosure form (available at https://tp.amegroups. com/article/view/10.21037/tp-21-11/coif). The authors have no conflicts of interest to declare.

Ethical Statement: The authors are accountable for all aspects of the work in ensuring that questions related to the accuracy or integrity of any part of the work are appropriately investigated and resolved. All procedures performed in studies involving human participants were in accordance with the ethical standards of the institutional and/or national research committee(s) and with the Helsinki Declaration (as revised in 2013). Written informed consent was obtained from the patient's legal guardian/next of kin for publication of this case report and accompanying images. A copy of the written consent is available for review by the editorial office of this journal.

Open Access Statement: This is an Open Access article distributed in accordance with the Creative Commons Attribution-NonCommercial-NoDerivs 4.0 International License (CC BY-NC-ND 4.0), which permits the noncommercial replication and distribution of the article with the strict proviso that no changes or edits are made and the original work is properly cited (including links to both the formal publication through the relevant DOI and the license). See: https://creativecommons.org/licenses/by-nc-nd/4.0/.

\section{References}

1. Reynen K. Cardiac myxomas. N Engl J Med 1995;333:1610-7.

2. Sawaya JI, Dakik HA. Angiographic visualization of an atrial myxoma. N Engl J Med 2000;342:294-5.

3. Bakkali A, Sedrati M, Cheikhaoui Y, et al. Cardiac myxomas (a series of 23 cases). Ann Cardiol Angeiol (Paris) 2009;58:94-8.

4. Jean WC, Walski-Easton SM, Nussbaum ES. Multiple intracranial aneurysms as delayed complications of an atrial myxoma: case report. Neurosurgery 2001;49:200-2; discussion 202-3.

5. Waliszewska-Prosół M, Zimny A, Chojdak-Łukasiewicz J, et al. Multiple fusiform cerebral aneurysms detected after atrial myxoma resection: a report of two cases. Kardiol Pol 2018;76:1571.

6. Mattle HP, Maurer D, Sturzenegger M, et al. Cardiac myxomas: a long term study. J Neurol 1995;242:689-94.

7. Pinede L, Duhaut P, Loire R. Clinical presentation of left atrial cardiac myxoma. A series of 112 consecutive cases.
Medicine (Baltimore) 2001;80:159-72.

8. De Marco A, La Tessa G, De Falco A, et al. Multiple Cerebral Aneurysms Associated with Atrial Myxoma: A Case Report. Neuroradiol J 2010;23:62-8.

9. Sandok BA, von Estorff I, Giuliani ER. Subsequent neurological events in patients with atrial myxoma. Ann Neurol 1980;8:305-7.

10. Gupta MM, Agrawal N. Oncotic cerebral aneurysms in a case of left atrial myxoma, role of imaging in diagnostics and treatment. Pol J Radiol 2015;80:490-5.

11. Rodriguez FJ, Brown RD, Mohr JP, et al. Embolic atrial myxoma with neoplastic aneurysm formation and haemorrhage: a diagnostic challenge. Neuropathol Appl Neurobiol 2006;32:213-6.

12. Budzilovich G, Aleksic S, Greco A, et al. Malignant cardiac myxoma with cerebral metastases. Surg Neurol 1979;11:461-9.

13. Kvitting JP, Engvall J, Broqvist M, et al. Recurrence of myxoma in the left ventricle with concurrent cerebral fusiform aneurysms after previous atrial myxoma surgery. J Thorac Cardiovasc Surg 2008;135:1172-3.

14. Yilmaz MB, Akin Y, Güray U, et al. Late recurrence of left atrial myxoma with multiple intracranial aneurysms. Int J Cardiol 2003;87:303-5.

15. Zheng J, Zhang J. Neoplastic cerebral aneurysm from metastatic tumor: a systematic review of clinical and treatment characteristics. Clin Neurol Neurosurg 2015;128:107-11.

16. Friedman DP, Rapoport RJ. Giant fusiform oncotic aneurysm: MR and angiographic findings. AJR Am J Roentgenol 1996;167:538-9.

17. Nucifora PG, Dillon WP. MR diagnosis of myxomatous aneurysms: report of two cases. AJNR Am J Neuroradiol 2001;22:1349-52.

18. Herbst M, Wattjes MP, Urbach H, et al. Cerebral embolism from left atrial myxoma leading to cerebral and retinal aneurysms: a case report. AJNR Am J Neuroradiol 2005;26:666-9.

19. Hwang BJ, Connelly MM, Lev MH. Distinctive MR imaging appearance of hemorrhagic cerebral aneurysms associated with atrial myxoma. AJR Am J Roentgenol 2001;177:925-7.

20. New PF, Price DL, Carter B. Cerebral angiography in cardiac myxoma. Correlation of angiographic and histopathological findings. Radiology 1970;96:335-45.

21. Ashalatha R, Moosa A, Gupta AK, et al. Cerebral aneurysms in atrial myxoma: a delayed, rare manifestation. Neurol India 2005;53:216-8. 
22. Radoi MP, Stefanescu F, Arsene D. Brain metastases and multiple cerebral aneurysms from cardiac myxoma: case report and review of the literature. Br J Neurosurg 2012;26:893-5.

23. Namura O, Saitoh M, Moro H, et al. A case of biatrial multiple myxomas with glandular structure. Ann Thorac Cardiovasc Surg 2007;13:423-7.

24. Sedat J, Chau Y, Dunac A, et al. Multiple cerebral aneurysms caused by cardiac myxoma: a case report and present state of knowledge. Interv Neuroradiol 2007;13:179-84.

25. Furuya K, Sasaki T, Yoshimoto Y, et al. Histologically verified cerebral aneurysm formation secondary to embolism from cardiac myxoma: case report. J Neurosurg 1995;83:170-3.

26. Orlandi A, Ciucci A, Ferlosio A, et al. Increased expression and activity of matrix metalloproteinases characterize embolic cardiac myxomas. Am J Pathol 2005;166:1619-28.

27. Roeltgen DP, Weimer GR, Patterson LF. Delayed neurologic complications of left atrial myxoma. Neurology 1981;31:8-13.

28. Stock K. Multiple cerebral aneurysms in a patient with recurrent cardiac myxomas: a case report. Interv Neuroradiol 2004;10:335-40.

29. Josephson SA, Johnston SC. Multiple stable fusiform intracranial aneurysms following atrial myxoma. Neurology 2005;64:526.

30. Zheng J, Li S, Cao Y, et al. Multiple cerebral myxomatous aneurysms: what is the optimal treatment? J Stroke Cerebrovasc Dis 2015;24:232-8.

31. Chiang KH, Cheng HM, Chang BS, et al. Multiple cerebral aneurysms as manifestations of cardiac myxoma: Brain imaging, digital subtraction angiography, and echocardiography. Tzu Chi Med J 2011;23:63-5.

32. Bernet F, Stulz PM, Carrel TP. Long-term remission after resection, chemotherapy, and irradiation of a metastatic

Cite this article as: Wang A, Wang Z, Hu K, Liu J, Cao Q, Zhang Z, Si Z. Delayed multiple intracranial aneurysms caused by left atrial myxoma: a case report and literature review. Transl Pediatr 2022;11(1):149-156. doi: 10.21037/tp-21-11 myxoma. Ann Thorac Surg 1998;66:1791-2.

33. Penn DL, Lanpher AB, Klein JM, et al. Multimodal treatment approach in a patient with multiple intracranial myxomatous aneurysms. J Neurosurg Pediatr 2018;21:315-21.

34. Kesav P, John S, Joshi P, et al. Cardiac Myxoma Embolization Causing Ischemic Stroke and Multiple Partially Thrombosed Cerebral Aneurysms. Stroke 2021;52:e10-4.

35. Branscheidt M, Frontzek K, Bozinov O, et al. Etoposide/carboplatin chemotherapy for the treatment of metastatic myxomatous cerebral aneurysms. J Neurol 2014;261:828-30.

36. Zhang S, Zhang Q, Yu H, et al. Neuroimaging characteristics and long-term prognosis of myxoma-related intracranial diseases. Neuroradiology 2020;62:307-17.

37. Branch CL Jr, Laster DW, Kelly DL Jr. Left atrial myxoma with cerebral emboli. Neurosurgery 1985;16:675-80.

38. Santillan A, Knopman J, Patsalides A, et al. Delayed intracranial aneurysm formation after cardiac myxoma resection: Report of two cases and review of the literature. Interv Neuroradiol 2019;25:177-81.

39. Garcia-Ptacek S, Matias-Guiu JA, Valencia-Sánchez C, et al. Mechanical endovascular treatment of acute stroke due to cardiac myxoma. J Neurointerv Surg 2014;6:e1.

40. Vega RA, Chan JL, Anene-Maidoh TI, et al. Mechanical thrombectomy for pediatric stroke arising from an atrial myxoma: case report. J Neurosurg Pediatr 2015;15:301-5.

41. Chen HJ, Liou CW, Chen L. Metastatic atrial myxoma presenting as intracranial aneurysms with hemorrhage: case report. Surg Neurol 1993;40:61-4.

42. Chen Z, Wang Y, Ye W, et al. Multiple Intracranial Aneurysms as Delayed Complication of Atrial Myxoma: Case Report and Literature Review. Interv Neuroradiol 2005;11:251-4. 